Published in final edited form as:

Aging Clin Exp Res. 2018 February ; 30(2): 209-212. doi:10.1007/s40520-017-0771-2.

\title{
Motivators for Alzheimer's Disease Clinical Trial Participation
}

\author{
Shoshana H. Bardach ${ }^{a, b}$, Sarah D. Holmes ${ }^{c}$, and Gregory A. Jichab,d \\ aGraduate Center for Gerontology, College of Public Health, University of Kentucky, Lexington, KY \\ bSanders-Brown Center on Aging, University of Kentucky, Lexington, KY \\ 'University of Maryland, Baltimore, MD \\ ${ }^{\mathrm{d} D e p a r t m e n t}$ of Neurology, University of Kentucky, Lexington, KY
}

\begin{abstract}
Background-Alzheimer's disease (AD) research progress is impeded due to participant recruitment challenges. This study seeks to better understand, from the perspective of individuals engaged in clinical trials (CTs), research motivations.

Methods-Participants, or their caregivers, from AD treatment and prevention CTs were surveyed about research motivators.

Results-The eighty-seven respondents had a mean age of 72.2, were predominantly Caucasian, $55.2 \%$ were male, and $56.3 \%$ had cognitive impairment. An overwhelming majority rated the potential to help themselves or a loved one and the potential to help others in the future as important motivators. Relatively few respondents were motivated by free healthcare, monetary rewards, or to make others happy.
\end{abstract}

Conclusions-Recruitment efforts should focus on the potential benefit for the individual, their loved ones, and others in the future rather than free healthcare or monetary rewards.

\section{Keywords}

Clinical Trials; Motivation; Recruitment

\footnotetext{
Corresponding Author: Shoshana H. Bardach, PhD, 1030 S. Broadway, Suite 5, Lexington, KY 40504, shbardach@uky.edu. Contributions

S. H. Bardach analyzed the data and wrote the paper. S. D. Holmes and G. A. Jicha planned the study, provided insights into analysis, and contributed to revising the paper.

Compliance with Ethical Standards

Conflict of Interest: The authors declare that they have no conflict of interest.

Ethical Approval: All procedures performed in studies involving human participants were in accordance with the ethical standards of the institutional and/or national research committee and with the 1964 Helsinki declaration and its later amendments or comparable ethical standards.

Informed Consent: Informed consent was obtained from all individual participants included in the study.

Data availability: The datasets during and/or analyzed during the current study available from the corresponding author on reasonable request.
} 


\section{Introduction}

Today over five million Americans have Alzheimer's disease (AD) and it is estimated that someone develops AD every 67 seconds in the United States. It is expected that by 2050 there will be between 11 and 16 million individuals living with AD in the United States if we do not experience any breakthroughs in therapeutic development [1]. While symptomatic treatments are currently available, at present there are no options for disease modifying therapies that might alter the disease course. Researchers are working to develop new medications or strategies to prevent or treat $\mathrm{AD}$, but recruitment of research participants remains a challenge [2].

Recruitment has historically been one of the largest impediments to successful clinical trials [3]. Currently, less than one third of trials achieve their initial recruitment target and even among those trials that do reach their desired participation numbers, half require a prolonged recruitment period [4]. Improving the science behind recruitment for AD clinical trials is imperative for advancing the rate of scientific discoveries for prevention and treatment [5]. Challenges to the recruitment of AD clinical trials include fear of medication side-effects, perceived lack of personal benefit of placebo-controlled trials and in prevention studies, fear over invasive procedures, the requirement of a study partner, logistical concerns such as time and travel, general skepticism towards research, and for treatment focused trials the underdiagnosis of $\mathrm{AD}$ and mild cognitive impairment [6]. While knowledge of these challenges can help inform study design to make trials more appealing to participants, addressing barriers does not guarantee that someone will chose to get involved. Much of the existing research exploring recruitment for clinical trials focuses on hypothetical research studies rather than real research participants [7]. In order to truly understand how to get individuals involved we need to better understand the motivations of participants who make the decision to enroll. Accordingly the purpose of this study is to better understand, from the perspective of individuals actually engaged in $\mathrm{AD}$ prevention and treatment clinical trials, motivations for research participation.

\section{Methods}

Surveys were mailed to 171 participants or their study partners from seven different AD prevention or treatment clinical trials being conducted at a single AD center. These studies included two for individuals with $\mathrm{AD}$, two for individuals with mild cognitive impairment (MCI) or $\mathrm{AD}$, one only for individuals with mild cognitive impairment, one for individuals with normal cognition or mild cognitive impairment, and one only for those with normal cognition. Surveys were mailed to active study participants, those who enrolled in one of the studies did not pass the screening process, and those who withdrew from one of the studies but agreed to be contacted about additional research. The surveys included a series of questions about potential motivators for research participation (e.g. to help themselves, to help others, to get free healthcare) and participants were asked to rate each item on a fivepoint Likert scale from "not at all important" to "extremely important". Responses were dichotomized to distinguish participants who rated a motivator as somewhat or extremely important from those that did not view that motivator as important. Analysis involved descriptive statistics to describe characteristics of the sample. Independent samples t-tests 
and chi-square tests were done to explore differences based on group characteristics (e.g. respondents and non-respondents, normal and impaired cognition, women and men). In addition, Mann-Whitney tests were used to examine the non-dichotomized ordinal responses.

\section{Results}

Surveys were completed by 87 of the 171 respondents, representing a $51.2 \%$ response rate. There were no significant differences between respondents and non-respondents in terms of cognitive status, sex, race, or age (see Table 1).

The majority of surveys were completed by the research participant themselves (78.2\%), with an average age of 72.4 (range of 54.6-89.8), just over half were male (55.2\%), most were Caucasian (87.4\%), and just over half were in a study for participants with cognitive impairment (MCI or AD) (56.3\%) (See Table 2).

There were no significant differences between those with normal and impaired cognition in terms of sex, race, or age.

An overwhelming majority of respondents rated the potential to help themselves or a loved one (92.9\%) and the potential to try to help others in the future (96.5\%) as important motivators for research participation. Relatively few respondents were motivated by free healthcare services (23\%) or monetary rewards $(4.6 \%)$. A minority of participants indicated being motivated to make a family member $(26.7 \%)$ or doctor $(15.1 \%)$ happy (see Figure 1 ).

Women $(38.5 \%)$ were significantly more likely than men (17.0\%) to report participating in research to make family members happy, $\mathrm{p}=.025$. There were no significant differences between those with normal and impaired cognition for any of the potential research motivators. There were also no significant age or racial differences in motivational factors based on whether the participant or study partner completed the survey.

Using Mann-Whitney statistics to explore the ranked responses, there were no statistically significant differences in motivational factors for participating based on the cognitive status or the race of the participant. Consistent with the dichotomized results, women rated the likelihood of participating to make a family member happy as more important than men, Mann-Whitney $U=697.0, n_{1}=47 n_{2}=39, P=.049$, two-tailed. Women also rated the likelihood of participating to make a doctor happy as more important than men, MannWhitney $U=668.5, n_{1}=47 n_{2}=39, P=.025$, two-tailed (see Figure 2).

Finally, those who completed the survey themselves rated the desire to help themselves as lower than those who completed the survey on behalf of the research participant, MannWhitney $U=364.0, n_{1}=66 n_{2}=16, P=.024$ two-tailed.

\section{Discussion}

The results of this study suggest that recruitment efforts for AD prevention and treatment clinical trials should focus on the potential benefit for the individual, their loved ones, and 
others in the future rather than personal benefits such as free healthcare or monetary rewards. Efforts should be made to convey the message that research has the potential to help not only the individual involved, but also to help others in the future. The potential to help others in the future may be a particularly critical message to convey as many AD prevention and treatment research studies provide no clear immediate personal benefit, yet still entail invasive study procedures such as spinal fluid collection.

In addition, these findings suggest that future recruitment efforts should explore ways to leverage social awareness and the need to promote research participation, particularly among women. Given that most AD prevention and treatment research includes a study partner, efforts to engage various care partners and life companions, including spouses, adult children, partners or friends, and promote understanding of the importance of research participation across these individuals and across ages and racial groups may strengthen both recruitment and retention efforts [8]. Potential study partners may encourage their loved ones to participate in research based on the hope it will help their loved one directly. While the potential benefits of the treatments or interventions under investigation cannot be guaranteed, efforts to promote additional recruitment could highlight other possible benefits that participants may receive, including the close medical monitoring of individuals engaged in research studies and the personal fulfillment of playing a valuable role in the search for better ways to prevent or treat $\mathrm{AD}$.

While this research was limited by a small sample size, one strength of this study is that responses come from research participants actually engaged in AD research, not from those being asked about hypothetical reasons they may choose to participate in research studies. The small sample size, however, may have prevented us from detecting differences in motivation for research engagement between different groups of participants. Future research should involve a larger, multi-site sample to explore whether motivations to participate in $\mathrm{AD}$ research may differ between participants and caregivers. Identifying potential differences in motivation along the cognitive continuum of participants may help to craft messages and target recruitment materials more effectively. While prior research has indicated how sociodemographic factors impact clinical trial eligibility, more work is needed to understand how these factors relate to willingness to participate [9]. Future research should gather a wider range of sociodemographic factors such as educational attainment and social support to better understand the nuances of research engagement. In addition, a larger sample should focus on expanding knowledge about the motivations for research participation among minority populations, who are largely underrepresented in AD research [10].

\section{Acknowledgments}

The authors thank all study participants and study partners who participate in research at our site and across the globe and particularly thank those who took the time to complete this survey.

Funding details

The authors have no funding to acknowledge for this research. 


\section{References}

1. Alzheimer's Association. 2015 Alzheimer's disease facts and figures. Alzheimers Dement. 2015; 11(3):332-84. [PubMed: 25984581]

2. Vellas B, et al. Alzheimer's disease therapeutic trials: EU/US Task Force report on recruitment, retention, and methodology. J Nutr Health Aging. 2012; 16(4):339-45. [PubMed: 22499454]

3. Knebl JA, Patki D. Recruitment of subjects into clinical trials for Alzheimer disease. J Am Osteopath Assoc. 2010; 110(9 Suppl 8):S43-9. [PubMed: 20926743]

4. McDonald AM, et al. What influences recruitment to randomised controlled trials? A review of trials funded by two UK funding agencies. Trials. 2006; 7(1):1-8. [PubMed: 16542027]

5. Watson JL, et al. Obstacles and opportunities in Alzheimer's clinical trial recruitment. Health Aff (Millwood). 2014; 33(4):574-9. [PubMed: 24711317]

6. Grill JD, et al. Risk disclosure and preclinical Alzheimer's disease clinical trial enrollment. Alzheimers Dement. 2013; 9(3):356-359.e1. [PubMed: 23141383]

7. Treweek S, et al. Methods to improve recruitment to randomised controlled trials: Cochrane systematic review and meta-analysis. BMJ Open. 2013; 3(2)

8. Grill JD, Karlawish J. Addressing the challenges to successful recruitment and retention in Alzheimer's disease clinical trials. Alzheimers Res Ther. 2010; 2(6):34. [PubMed: 21172069]

9. Cooper C, Ketley D, Livingston G. Systematic review and meta-analysis to estimate potential recruitment to dementia intervention studies. International Journal of Geriatric Psychiatry. 2014; 29(5):515-525. [PubMed: 24706605]

10. Williams MM, et al. Barriers and facilitators of African American participation in Alzheimer disease biomarker research. Alzheimer Dis Assoc Disord. 2010; 24(Suppl):S24-9. [PubMed: 20711059] 


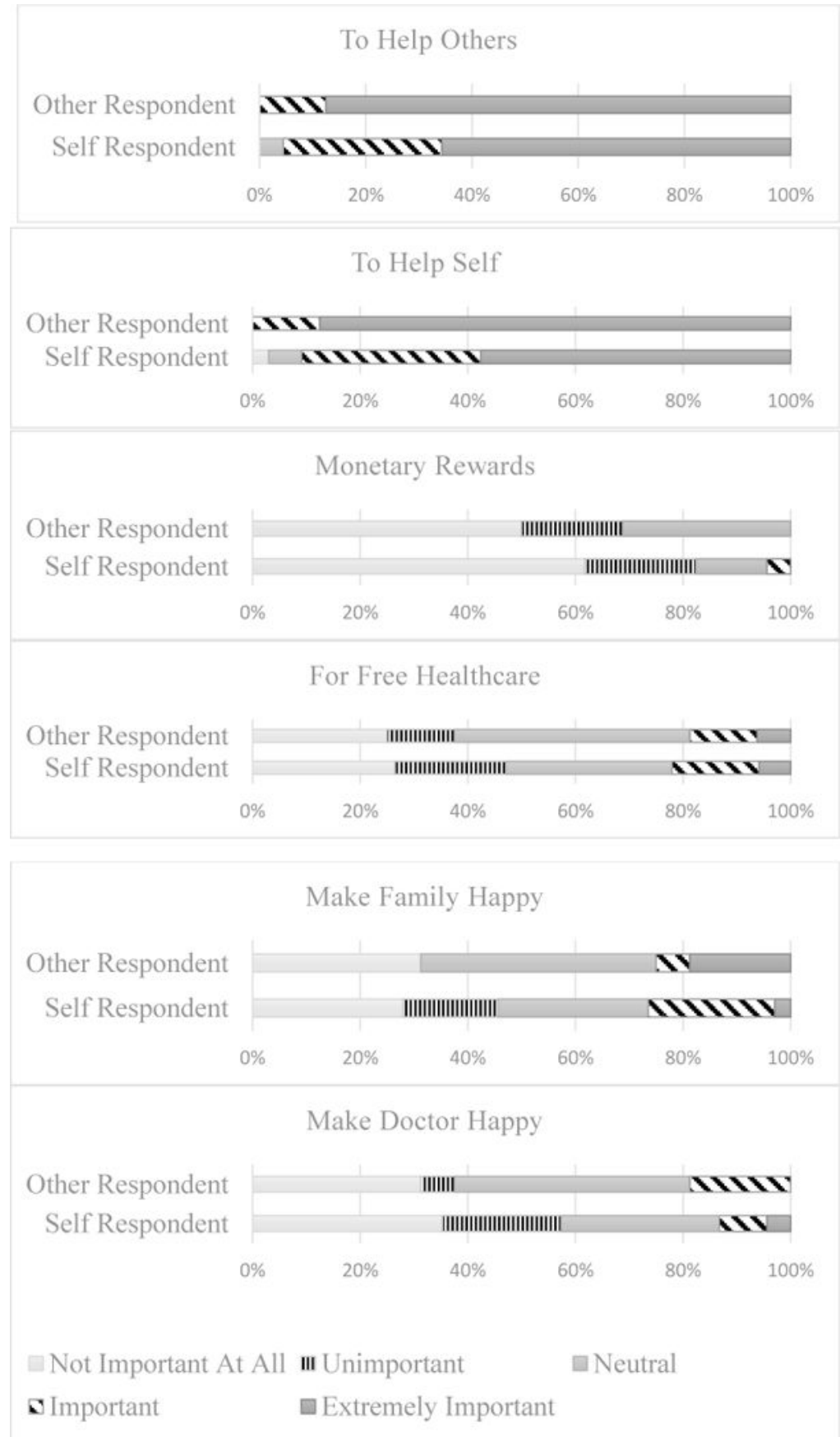

Figure 1.

Motivations for Participation - Self and Other Respondents

Aging Clin Exp Res. Author manuscript; available in PMC 2019 February 01. 
To Make a Family Member Happy

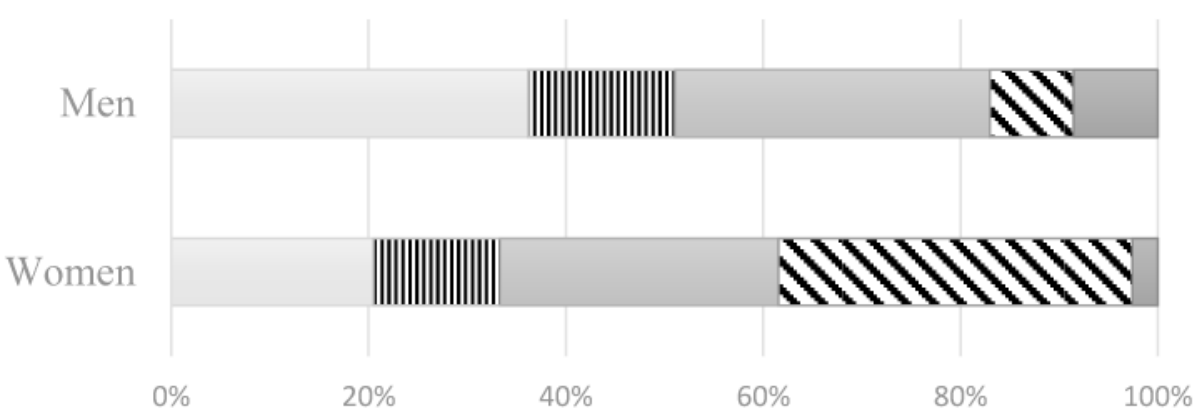
$\square$ Not Important At All
III Unimportant
$\square$ Neutral

- Important

$\square$ Extremely Important

To Make a Doctor Happy

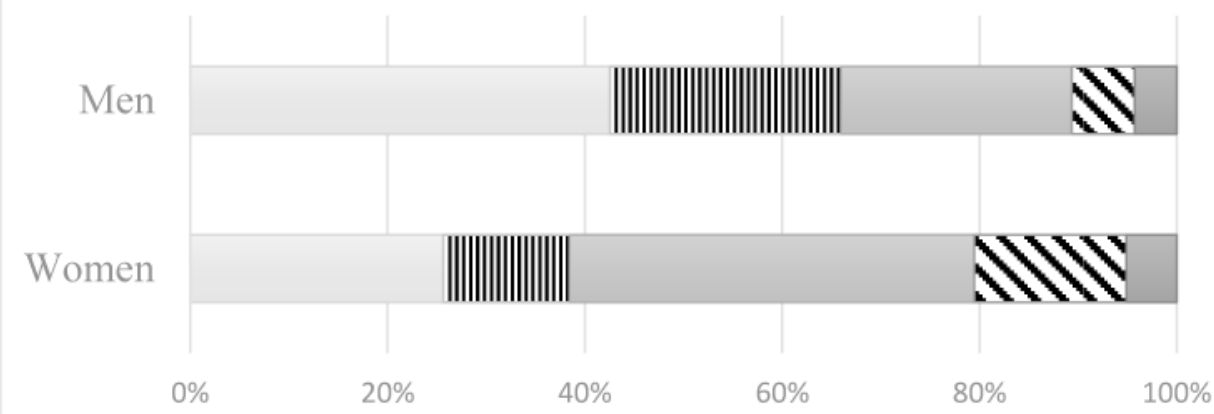
Not Important At All
III Unimportant
$\square$ Neutral
$\checkmark$ Important
Extremely Important

Figure 2.

Gender Differences in Research Motivations 


\section{Table 1}

Characteristics of Respondents and Non-Respondents

\begin{tabular}{|c|c|c|}
\hline & $\begin{array}{l}\text { Respondents } \\
\mathbf{n}=87, \mathbf{n}(\%)\end{array}$ & $\begin{array}{l}\text { Non-Respondents } \\
\mathbf{n = 8 3}, \mathbf{n}(\%)\end{array}$ \\
\hline \multicolumn{3}{|l|}{ Cognitive Status } \\
\hline Normal & $38(43.7)$ & $34(41.0)$ \\
\hline Impaired & $49(56.3)$ & $49(59)$. \\
\hline \multicolumn{3}{|l|}{$\operatorname{Sex}$} \\
\hline Male & $48(55.2)$ & $39(47.0)$ \\
\hline Female & $39(44.8)$ & $44(53.0)$ \\
\hline \multicolumn{3}{|l|}{ Race } \\
\hline Caucasian & $76(87.4)$ & $71(85.5)$ \\
\hline Other & $11(12.6)$ & $12(14.5)$ \\
\hline Age, mean (SD) & $72.4(7.4)$ & $73.2(7.2)$ \\
\hline
\end{tabular}


Table 2

Respondent Characteristics

\begin{tabular}{|c|c|c|c|}
\hline & $\begin{array}{l}\text { Normal Cognition } \\
\mathbf{n}=\mathbf{3 8}, \mathbf{n}(\%)\end{array}$ & $\begin{array}{l}\text { Impaired Cognition } \\
n=49, n(\%)\end{array}$ & $\begin{array}{l}\text { All Respondents } \\
\mathbf{n}=\mathbf{8 7}, \mathbf{n}(\%)\end{array}$ \\
\hline \multicolumn{4}{|l|}{ Respondent } \\
\hline Participant & 37 (97.4) & $31(63.3)$ & $68(78.2)$ \\
\hline Study Partner & $1(2.6)$ & 15 (30.6) & $16(18.4)$ \\
\hline Unknown & 0 & $3(6.1)$ & $3(3.4)$ \\
\hline \multicolumn{4}{|l|}{$\operatorname{Sex}$} \\
\hline Male & $20(52.6)$ & $28(57.1)$ & $48(55.2)$ \\
\hline Female & $18(47.4)$ & $21(42.9)$ & $39(44.8)$ \\
\hline \multicolumn{4}{|l|}{ Race } \\
\hline Caucasian & $32(84.2)$ & $44(89.8)$ & $76(87.4)$ \\
\hline Other & $6(15.8)$ & $5(10.2)$ & 11 (12.6) \\
\hline Age, mean (SD) & $72.2(4.5)$ & $72.5(9.1)$ & $72.4(7.4)$ \\
\hline
\end{tabular}

